\title{
Association of Ideal Cardiovascular Health with Carotid Intima-Media thickness (cIMT) in a Young Adult Population: Tehran Lipid and Glucose Study (TLGS)
}

\section{Vajihe Chavoshi}

Obesity Research Center, Research Institute for Endocrine Sciences, Shahid Beheshti University of Medical Sciences

\section{Maryam Barzin}

Obesity Research Center, Research Institute for Endocrine Sciences, Shahid Beheshti University of Medical Sciences

\section{Amir Ebadinejad}

Obesity Research Center, Research Institute for Endocrine Sciences, Shahid Beheshti University of

Medical Sciences

\section{Pooneh Dehghan}

Imaging Department, Taleghani Hospital, Shahid Beheshti University of Medical Sciences,

\section{Amin Momeni Moghaddam}

Imaging Department, Taleghani Hospital, Shahid Beheshti University of Medical Sciences,

\section{Maryam Mahdavi}

Obesity Research Center, Research Institute for Endocrine Sciences, Shahid Beheshti University of

Medical Sciences

\section{Farzad Hadaegh}

Prevention of Metabolic Disorders Research Center, Research Institute for Endocrine Sciences, Shahid Beheshti University of Medical Sciences,

\section{Mahtab Niroomand}

Endocrinology Division, Department of Internal Medicine, Shahid Beheshti University of Medical

Sciences

\section{Majid Valizadeh}

Obesity Research Center, Research Institute for Endocrine Sciences, Shahid Beheshti University of

Medical Sciences

\section{Fereidoun Azizi}

Endocrine Research Center, Research Institute for Endocrine Sciences, Shahid Beheshti University of Medical Sciences

Parvin Mirmiran 
Nutrition and Endocrine Research Center, Research Institute for Endocrine Sciences, Shahid Beheshti University of Medical Sciences

\section{Farhad Hosseinpanah ( $\sim$ fhospanah@endocrine.ac.ir)}

Obesity Research Center, Research Institute for Endocrine Sciences, Shahid Beheshti University of Medical Sciences

\section{Research Article}

Keywords: ideal cardiovascular health, cardiovascular risk, carotid intima-media thickness, subclinical cardiovascular disease

Posted Date: October 7th, 2021

DOl: https://doi.org/10.21203/rs.3.rs-952375/v1

License: (c) (1) This work is licensed under a Creative Commons Attribution 4.0 International License. Read Full License 


\section{Abstract}

Ideal cardiovascular health $(\mathrm{CVH})$ is associated with a lower risk of developing cardiovascular diseases. This study aims to investigate the association of $\mathrm{CVH}$ metrics with carotid intima-media thickness (cIMT) as a marker of subclinical atherosclerosis in young adults. A cross-sectional study was performed on 1295 adults, average age of $29.7 \pm 4.0$ years, selected among the participants of the Tehran Lipid and Glucose Study (TLGS). The participants were divided into two CVH groups: Ideal CVH and poor/intermediate $\mathrm{CVH}$. Multivariate-adjusted linear regression was used to determine the association of ideal CVH score with cIMT. Multivariate-adjusted odd ratios (ORs) were calculated for high cIMT ( $\geq 95 \%$ percentile). Also, the independent effects of each ideal CVH metric on clMT were analyzed. The prevalence of ideal $\mathrm{CVH}$ was $9.3 \%$ in the studied population, and the mean of cIMT was $0.55 \pm 0.09 \mathrm{~mm}$. A 1-point increase in $\mathrm{CVH}$ score was associated with a decrease of $0.128 \mathrm{~mm}$ (Beta [SE] $=-0.128$ [0. 002], $\mathrm{p}<0.001)$ in clMT and rendered an odd ratio of $0.68(\mathrm{OR}=0.68$ [95\% Cl: $0.56-0.82$ ], $\mathrm{p}<0.001)$ for having a high CIMT ( $\geq 95 \%$ percentile). Each ideal glucose, ideal blood pressure and ideal body mass index (BMI) had a significant inverse association with cIMT. There was a graded inverse association between ideal CVH score and cIMT among young adults, indicating that ideal CVH metrics are associated with better vascular health in this population. The low prevalence of ideal CVH highlighted the importance of implementing health promotion strategies.

\section{Introduction}

Cardiovascular diseases (CVD) are the leading causes of global mortality, with approximately 17.8 million deaths being reported due to diseases like coronary heart disease and stroke in just 2017 (1). More than three-quarters of CVD-related deaths occur in low- and middle-income countries (2). The American Heart Association presented the concept of ideal cardiovascular health (CVH) in 2010, intending to decrease CVD mortality by $20 \%$ in the following decade $(3,4)$. Cardiovascular health metrics include seven items, of which four are related to health behaviors (i.e., smoking status, body mass index, physical activity, and diet), and the other three are important health indicators (i.e., total cholesterol, blood pressure, and fasting blood glucose). According to studies, achieving an ideal CVH status is associated with lower CVD-related mortality and morbidity and better cardiovascular outcomes (5-7).

Subclinical atherosclerosis is an important predictor of CVD beyond traditional risk factors $(8,9)$. Carotid intima-media thickness (cIMT) is a common ultrasound-based measurement of arterial wall thickness used to evaluate atherosclerosis (10). This parameter has been shown to predict the risk of atherosclerotic plaque formation and CVD development in the future $(11,12)$. Numerous epidemiological studies have shown an association between ideal $\mathrm{CVH}$ and a reduction in the risk of subclinical cardiovascular diseases characterized by changes in cIMT $(6,13-16)$. Previously, evaluating the seven $\mathrm{CVH}$ metrics in Iranian men and women in the Tehran Lipid and Glucose Study (TLGS) indicated a low prevalence of ideal $\mathrm{CVH}$ in the adult population (17). Therefore, it is important to investigate a potential relationship between $\mathrm{CVH}$ metrics and the incidence of subclinical atherosclerosis to prevent 
cardiovascular events. Moreover, to our knowledge, there is no study in the Middle East and North Africa (MENA) region to assess the relationship between CVH metrics and cIMT.

This population-based study aimed to assess the association of the ideal $\mathrm{CVH}$ score and each of the seven $\mathrm{CVH}$ metrics, with the risk of developing subclinical atherosclerosis, defined by increased clMT, among Iranian young adults in the framework of the TLGS.

\section{Material And Methods}

\section{Study Participants and Design}

In this cross-sectional study, we used the data available from the TLGS, a cohort study initiated in 1998, to identify the risk factors of non-communicable diseases in Tehran urban populations. The details of this population-based study have been reported elsewhere (18). In the present study, we used the data collected in phase VI (2015-2018) of the TLGS. Among the subjects aged 20-40 years old and had already undergone routine evaluations in phase VI $(n=2641)$, a number of the participants were recruited for cIMT measurement $(n=1455)$. After excluding those with a BMI $<20 \mathrm{~kg} / \mathrm{m}^{2}$ at baseline $(n=77)$, the subjects using corticosteroids $(n=38)$, pregnant women $(n=13)$, those with a history of malignancies $(n=4)$, and individuals with distorted cIMT measurement $(n=5)$, a total number of 1295 participants were recruited for the current study (Figure 1).

\section{Measurements And Definitions}

Trained interviewers used standard questionnaires to obtain demographic data, smoking status, dietary intake, physical activity, medical history, and drug consumption history. Also, trained personnel performed anthropometric examinations. A digital electronic weighing scale (range: 0.1-150 kg, Seca 707, Hanover, MD, USA) was used to measure weight that was recorded to the nearest of $100 \mathrm{~g}$ with the participants being shoeless and minimally clothed. A tape meter was used to measure height in the standing position, and BMI was calculated as weight (in $\mathrm{Kg}$ ) divided by height (in squared meters) $\left(\mathrm{kg} / \mathrm{m}^{2}\right)$. Duplicate measurements (15-minute apart) of systolic and diastolic blood pressure were done by a qualified physician using a standard mercury sphygmomanometer applied on the seated participant's right arm. The mean of the two measurements was calculated and regarded as the subject's blood pressure. Venous blood samples were taken after overnight fasting of 12-14 h, centrifuged within 30-45 min of collection, and finally analyzed at the TLGS research laboratory. Details of measuring serum biochemistry parameters, FPG, and lipids have been reported elsewhere (18).

\section{Determining Cimt}

The participants underwent ultrasound examination using a linear 7.5-10 MHz transducer (Samsung Medison SonoAceR3 Ultrasound, South Korea). Two radiologists performed the examinations in the 
supine position with the neck extended and slightly rotated to the opposite side of the examination. The initial carotid scan was performed in the transverse plane throughout the artery to evaluate its anatomy, locate any atherosclerotic plaque, and determine the site of maximal wall-thickening. Measurements were done in plaque-free arterial segments fulfilling the optimal B-mode imaging criteria described below. A clear vision of the far arterial wall interface with a completely anechoic luminal content was considered to be an optimal greyscale carotid artery image and was saved for cIMT measurement. The IMT was defined as a hypoechoic band between the arterial wall's echogenic intimal and adventitial surfaces. The distance between the leading edge of the first and second echogenic lines of the far walls of the distal segment of the common carotid artery on both sides was measured at three locations, and the average was regarded as the final measurement of that side. Left common carotid artery (LCCA) far wall measurements were used for defining high cIMT. The degree of CIMT measures agreement between the two radiologists was evaluated by using an interclass correlation coefficient (ICC). ICC estimates and their $95 \%$ confident intervals were calculated using SPSS statistical package version 20 based on 2-way mixed-effects model and reported ICC results as ICC $=0.79$ with $95 \%$ confident interval $=0.55-0.90$. The ICC is a value between 0 and 1 , where values between 0.75 and 0.9 indicates good reliability (19). Moreover, the mean (SD) difference regarding between-rate ICC was 0.08 (0.12).

\section{Determining Cvh Status}

The AHA 2020 impact goals (3) were used to define cardiovascular health (CVH). Four ideal health behaviors (no smoking within the last year, ideal BMI, ideal physical activity, and an ideal diet) and three ideal health factors (untreated total cholesterol $<200 \mathrm{mg} / \mathrm{dL}$, untreated blood pressure $<120 / 80 \mathrm{~mm} \mathrm{Hg}$, and untreated glucose $<100 \mathrm{~mm} \mathrm{Hg}$ ) were considered to determine the $\mathrm{CVH}$ status of each individual. The number of ideal CVH metrics summarized the participants' CVH scores. An ideal CVH status was defined as having six or seven $\mathrm{CVH}$ metrics; intermediate $\mathrm{CVH}$ was defined as having 3-5 metrics, and poor $\mathrm{CVH}$ was regarded as having $0-2$ of the metrics. To ensure the presence of a sufficient number of participants within each group, we categorized them into two groups: those with six or more ideal CVH metrics $v s$. those with less than six ideal CVH metrics.

A checklist for dietary habits, a qualitative Food Frequency Questionnaire (FFQ), and two 24-hour dietary recall scales was used to assess the dietary status. The validity and reliability of the Persian translated version of FFQ had already been verified for evaluating the food intake status of the participants of the TLGS (20). In the present study, after excluding individuals with an extreme energy intake ( $\pm 3 S D)$, five AHA's ideal CV health components were used to calculate the participants' dietary scores ( $\geq 4.5$ cups per day of fruits and vegetables; $\geq 2$ to $3.5 \mathrm{oz}$ serving of fish per week; $\geq 31$-oz equivalents serving per day of whole grains; $<1500 \mathrm{mg}$ per day sodium; and $\leq 36$ oz per week of sugar-sweetened beverages [ $\leq 450 \mathrm{kcal}$ per week]) (3).

The Modifiable Activity Questionnaire (MAQ) was used to assess physical activity. The validity and reliability of the Persian translated version of MAQ had already been confirmed for evaluating the 
physical activity of TLGS participants (21). According to the minutes of vigorous or moderate physical activity, patients were classified into ideal ( $\geq 1500 \mathrm{~min} /$ week), intermediate (600-1500 min/week), and poor (<600 min/week) groups.

\section{Statistical Analysis}

The number of ideal CVH metrics was described for the participants. Normally distributed and skewed continuous variables were illustrated as Mean $\pm S D$ and median (IQ 25-75), respectively. Categorical variables were reported by the frequency (percentage) statistic. Quantitative variables with normal distribution were analyzed by the independent t-test, and quantitative variables with skewed distribution were analyzed using the Mann-Whitney $U$ test. Qualitative variables were analyzed using the Chi-square test, when appropriate. Multiple linear regression was used to assess the independent effects of the ideal $\mathrm{CVH}$ metrics on cIMT, adjusting for age, sex, and each metric separately, and then in a mutually adjusted model for all seven ideal CVH metrics. Odds for having a significantly high cIMT (cIMT > 95 percentile) were also estimated using logistic regression models. To handle missing values, the multiple imputations by chained equations (MICE) method was employed. All the analyses were performed in STATA version 12 SE (STATA Inc., TX, USA), considering a two-tailed P value of $<0.05$ as statistically significant.

\section{Results}

The study participants $(n=1295)$ aged $29.7 \pm 4.0$ years old, and $51.7 \%$ of them were men. The means of BMl and cIMT were $26.2 \pm 4.8 \mathrm{~kg} / \mathrm{m}^{2}$ and $0.55 \pm 0.09 \mathrm{~mm}$, respectively. Other characteristics of the studied population have been illustrated in Table 1. Regarding the $\mathrm{CVH}$ status, the participants were divided into three distinct groups (Figure 2). The majority of the participants had intermediate $\mathrm{CVH}(\mathrm{n}=1039,80.2 \%)$, followed by poor $\mathrm{CVH}(\mathrm{n}=135,10.4 \%)$, and then ideal $\mathrm{CVH}(\mathrm{n}=121,9.3 \%)$. 
Table 1

Descriptive Characteristics of the participants

\begin{tabular}{|ll|}
\hline Variable & Mean \pm SD or $\mathbf{n}(\%)$ \\
\hline Number & 1295 \\
\hline Age (year) & $29.7 \pm 4.0$ \\
\hline Men n (\%) & $670(51.7)$ \\
\hline BMI (kg/m2) & $26.2 \pm 4.8$ \\
\hline SBP $(\mathrm{mm} \mathrm{Hg})$ & $107.1 \pm 12.2$ \\
\hline DBP (mm Hg) & $72.8 \pm 9.3$ \\
\hline Total cholesterol (mg/dl) & $172.1 \pm 34.0$ \\
\hline Glucose (mg/dl) & $88.4 \pm 9.7$ \\
\hline Healthy diet score & $1.7 \pm 0.8$ \\
\hline Ideal smoking n (\%) & 967 (74.7) \\
\hline Ideal physical activity (min/week) & 581 (44.9) \\
\hline clMT, (mm) & $0.55 \pm 0.09$ \\
\hline $\begin{array}{l}\text { BMl, Body Mass Index; SBP, Systolic Blood Pressure; DBP, Diastolic Blood Pressure; clMT, Carotid } \\
\text { Intima-Media thickness }\end{array}$ \\
\hline
\end{tabular}

Table 2 shows the characteristics of the study participants according to different $\mathrm{CVH}$ groups. To ensure the presence of a sufficient number of subjects per group, those with poor/intermediate CVH ( $\leq$ five ideal CVH metrics, $\mathrm{n}=1174$ ) were compared with individuals with ideal $\mathrm{CVH}$ (six or seven ideal CVH metrics, $n=121)$. The two groups of the participants showed a similar average age $(29.6 \pm 4.1 \mathrm{vs} .30 .4 \pm 3.9$, $\mathrm{p}=0.074)$ and cIMT $(0.55 \pm 0.09$ vs. $0.55 \pm 0.09, \mathrm{p}=0.39)$ (Figure 3). Mean BMI, SBP, DBP, total cholesterol, FBS, and ideal diet score were higher in the poor/intermediate CVH group that also covered a higher ratio of smokers $(27.9 \%$ vs. $0.8 \%, \mathrm{p}<0.001)$. The median duration of moderate or vigorous physical activity was significantly lower in the poor/intermediate CVH group (1065.1 [347.3 - 2697.9] vs. 3000.7 [2072.7 5559.2], $\mathrm{p}<0.001$ ). 
Table 2

Baseline Characteristics According to $\mathrm{CVH}$ status

\begin{tabular}{|c|c|c|c|}
\hline Variable & $\begin{array}{l}\text { Poor \& Intermediate CVH } \\
(n=1174)\end{array}$ & $\begin{array}{l}\text { Ideal CVH } \\
(\mathrm{n}=121)\end{array}$ & $\begin{array}{l}\text { P- } \\
\text { Value }\end{array}$ \\
\hline Age, (year) & $29.8 \pm 4.0$ & $29.5 \pm 4.1$ & 0.438 \\
\hline Male, n (\%) & $627(53.4)$ & $43(35.5)$ & $<0.001$ \\
\hline BMI, (kg/m2) & $26.6 \pm 4.8$ & $21.8 \pm 2.3$ & $<0.001$ \\
\hline SBP, $(\mathrm{mm} \mathrm{Hg})$ & $107.9 \pm 12.1$ & $98.7 \pm 9.6$ & 0.006 \\
\hline DBP, (mm Hg) & $73.4 \pm 9.3$ & $66.9 \pm 6.8$ & 0.002 \\
\hline Total cholesterol, (mg/dl) & $173.6 \pm 34.6$ & $157.5 \pm 23.2$ & $<0.001$ \\
\hline Fasting glucose, (mg/dl) & $88.8 \pm 9.9$ & $84.6 \pm 6.1$ & 0.005 \\
\hline Current smoker, n (\%) & 327 (27.9) & $1(0.8)$ & $<0.001$ \\
\hline physical activity (min/week) & $1065.1(347.3,2697.9)$ & $3000.7(2072.7,5559.2)$ & $<0.001$ \\
\hline Ideal diet score & $1.7 \pm 0.8$ & $1.7 \pm 0.9$ & 0.155 \\
\hline $\begin{array}{l}\text { Family history of premature } \\
\text { CVD } n(\%)\end{array}$ & $40(3.4)$ & $5(4.1)$ & 0.604 \\
\hline $\begin{array}{l}\text { Educational level > } 12 \text { years, } n \\
(\%)\end{array}$ & $684(58.3 \%)$ & $73(60.3)$ & 0.699 \\
\hline clMT, (mm) & $0.55 \pm 0.09$ & $0.55 \pm 0.09$ & 0.398 \\
\hline \multicolumn{4}{|c|}{$\begin{array}{l}\text { BMI, Body Mass Index; SBP, Systolic Blood Pressure; DBP, Diastolic Blood Pressure; HDL, High-Density } \\
\text { Lipoprotein cholesterol; BMI, Body Mass Index; cIMT, Carotid Intima-Media thickness; CVD, } \\
\text { cardiovascular disease }\end{array}$} \\
\hline \multicolumn{4}{|c|}{$\begin{array}{l}\text { Data are presented as mean } \pm \text { SD or } n(\%) \text { except minutes of moderate or vigorous physical } \\
\text { activity/week which are presented as median (IQ 25-75). }\end{array}$} \\
\hline
\end{tabular}

Table 3 shows the independent effects of each ideal CVH metric on cIMT (in mm). In the model adjusted for age and sex (Model I), three ideal metrics were significantly associated with a higher cIMT: ideal blood pressure (Beta [SE] $=-0.124$ [0.006], $p<0.001)$, ideal glucose $(-0.060$ [0.010], $p=0.029)$, ideal BMI $(-0.101[0.005], p<0.001)$. A 1-point increase in the CVH score was also associated with a decrease of $0.128 \mathrm{~mm}$ in cIMT (Beta [SE] = -0.128 [0. 002], $\mathrm{p}<0.001$ ). The results did not change in Model II, which was mutually adjusted for all the variables of age, sex, family history of premature CVD, and educational level. 
Table 3

Independent Effects of Ideal Cardiovascular Health Metrics on cIMT(in millimeter)

\begin{tabular}{|lllll|}
\hline variable & Model I & & Model II & \\
\hline Ideal blood pressure & Beta(SE) & P-value & Beta(SE) & P-value \\
\hline Ideal total cholesterol & $-0.124(0.006)$ & $<0.001$ & $-0.124(0.006)$ & $<0.001$ \\
\hline Ideal glucose & $-0.002(0.007)$ & 0.942 & $-0.003(0.007)$ & 0.922 \\
\hline Nonsmoking & $-0.060(0.010)$ & 0.029 & $-0.059(0.010)$ & 0.032 \\
\hline Ideal BMI & $-0.009(0.006)$ & 0.763 & $-0.008(0.006)$ & 0.769 \\
\hline Ideal diet & $-0.101(0.005)$ & $<0.001$ & $-0.100(0.005)$ & $<0.001$ \\
\hline Ideal physical activity & $0.035(0.021)$ & 0.199 & $0.035(0.021)$ & 0.204 \\
\hline Ideal CVH Score & $-0.043(0.005)$ & 0.115 & $-0.045(0.005)$ & 0.103 \\
\hline $\begin{array}{l}\text { BMI, Body Mass Index; CVH, Cardiovascular Health; CVD, cardiovascular disease } \\
\text { Model I adjusted for age, sex }\end{array}$ & $-0.128(0.002)$ & $<0.001$ & $-0.128(0.002)$ & $<0.001$ \\
\hline $\begin{array}{l}\text { Model II mutually adjusted for all variables and for age, sex, family history of premature CVD, } \\
\text { Educational level }\end{array}$ & & & \\
\hline
\end{tabular}

Table 4 displays the independent predictive value of each ideal CVH metric (odd ratio, 95\% confidence interval) for having a high cIMT ( $>95$ percentile). In a fully adjusted model, two metrics inversely correlated with high cIMT: ideal blood pressure (OR [95\% Cl] $=0.31$ [0.19-0.51], $\mathrm{p}<0.001)$ and ideal BMI (0.46 [0.27-0.77], $p=0.003)$. A 1-point increase in the $\mathrm{CVH}$ score also predicted a decrease in the probability of having a high cIMT $(0.68[0.56-0.82], \mathrm{p}<0.001)$. 
Table 4

Independent Effects of Ideal Cardiovascular Health Metrics on cIMT (95 percentile)

\begin{tabular}{|lllll|}
\hline Variable & Model I & & Model II & \\
\hline Ideal blood pressure & OR(CI) & P-value & OR(CI) & P-value \\
\hline Ideal total cholesterol & $0.31(0.19,0.51)$ & $<0.001$ & $0.31(0.19,0.51)$ & $<0.001$ \\
\hline Ideal glucose & $0.87(0.49,1.53)$ & 0.637 & $0.87(0.49,1.53)$ & 0.640 \\
\hline Nonsmoking & $0.58(0.28,1.22)$ & 0.156 & $0.58(0.27,1021)$ & 0.149 \\
\hline Ideal BMI & $1.55(0.83,2.87)$ & 0.162 & $1.55(0.83,2.87)$ & 0.162 \\
\hline Ideal diet & $0.47(0.28,0.78)$ & 0.004 & $0.46(0.27,0.77)$ & 0.003 \\
\hline Ideal physical activity & $1.59(0.36,6.99)$ & 0.535 & $1.62(0.36,7.11)$ & 0.52 \\
\hline Ideal CVH Score & $0.64(0.39,1.03)$ & 0.067 & $0.64(0.40,1.04)$ & 0.072 \\
\hline $\begin{array}{l}\text { BMI, Body Mass Index; } \mathrm{CVH} \text {, Cardiovascular Health; CVD, cardiovascular disease } \\
\text { Model I adjusted for age, sex }\end{array}$ & $0.68(0.56,0.82)$ & $<0.001$ & $0.68(0.56,0.82)$ & $<0.001$ \\
\hline $\begin{array}{l}\text { Model II mutually adjusted for all variables and for age, sex, family history of premature CVD } \\
\text {,Educational level }\end{array}$ & & & \\
\hline
\end{tabular}

\section{Discussion}

In this population-based study that was conducted in the framework of the TLGS, the association of the ideal $\mathrm{CVH}$ score and each of the seven $\mathrm{CVH}$ metrics with cIMT was evaluated. The participants in this study had a mean age of 30 years, and the prevalence of ideal $\mathrm{CVH}$ among them was 9.3\%. A 1-point increase in the CVH score was associated with a decrease of $0.128 \mathrm{~mm}$ in cIMT and decreased the probability of presenting with a high cIMT ( $>95$ percentile) by $32 \%$. Each ideal glucose, ideal blood pressure, and ideal BMI had a significant inverse association with cIMT. Also, having ideal blood pressure and ideal BMI factors reduced the chances of developing high cIMT by $69 \%$ and $53 \%$, respectively.

Cardiovascular health metrics introduced by the AHA in the past decade to predict cardiovascular events consist of seven metrics (3). Based on studies, an ideal CVH status correlates with better cardiovascular outcomes $(5,7)$. The low prevalence of ideal $\mathrm{CVH}$ is an important global concern, especially in the middleand low-income countries (22). In our study, the prevalence of ideal CVH in a young adult Iranian population was $9.3 \%$. The prevalence of the ideal CVH status varies among studies, depending on populations' age and gender distribution and geographic variances (23). A systematic review of 88 studies reported that the prevalence of having five or more ideal CVH metrics was $19.6 \%$ (95\% Cl: $15.2 \%$ $23.9 \%$ ), and a poor CVH status was about twice in the elderly than in the young population (23). Previous studies have reported a low prevalence $(0.3-4 \%)$ of $\geq 6$ ideal $\mathrm{CVH}$ metrics in developing countries (5). 
Similarly, in the STEPwise study in Iran, although the prevalence of ideal CVH metrics among the population aged 20 to 65 years old reached about $7.2 \%$ in 2011, it again decreased to $<4 \%$ in 2016 (24).

The results of our study supported earlier studies demonstrating an inverse relationship between ideal CVH and CIMT (13-16). This is important as CIMT is a subclinical marker of atherosclerosis and a factor predisposing people to cardiovascular diseases $(5,11)$. We found that a 1-point increase in the CVH score was associated with a decline of $0.128 \mathrm{~mm}$ in CIMT and decreased the probability of presenting with a high cIMT after adjustment for age and sex. Nevertheless, the association of ideal CVH with cIMT did not change after further adjustments for the family history of premature CVD and educational level. The age range of our participants was between 20 and 40 years old. To our knowledge, there is only one crosssectional study on a similar population, in which five different cohorts of western populations were assessed, reporting that cIMT was $0.006 \mathrm{~mm}(95 \% \mathrm{Cl}$ : 0.012-0.003 $\mathrm{mm}$ ) thinner for each additional ideal $\mathrm{CVH}$ score (14). Likewise, other studies investigating the association between ideal CVH score and cIMT in adult populations in Spain, USA, and Africa revealed that a 1-point increase in the ideal CVH score was associated with $0.011,0.04$, and $0.005 \mathrm{~mm}$ cIMT reduction, respectively $(13,15,16)$. It is important to note that to our knowledge, there is only one longitudinal study conducted in China that evaluates the association between $\mathrm{CVH}$ metrics and cIMT. Wang et al.(25) after excluding individuals with elevated CIMT at the baseline, examined the association of CVH metrics with cIMT changes over approximately four years and showed that ideal CVH score were significantly and inversely related to the risk of developing subclinical atherosclerosis.

Ideal glucose, ideal blood pressure, and ideal BMI had a significant inverse association with cIMT. Similarly, Nonterah et al.(13) demonstrated an inverse association between the same ideal CVH metrics and CIMT in populations from four African countries. On the other hand, Oikonen et al. (14) indicated that the ideal status of each of blood pressure, BMI, cholesterol, and diet was independently and inversely associated with cIMT, whereas physical activity was directly associated with cIMT. According to these findings, differences in the weight of each of the seven metrics on cIMT should be considered when evaluating the effectiveness of the metrics.

The findings of this report are subjected to at least two limitations. First, it should be kept in mind that the observed inverse associations between ideal CVH metrics and cIMT were based on cross-sectional data, precluding the analysis of causal associations. The second limitation was that based on a previous study (16), the two groups of $\mathrm{CVH}$ were merged into one group due to sample size restrictions. As the main strength, this study is the first population-based report on the association of $\mathrm{CVH}$ metrics with cIMT in a young adult population in the MENA region. Also, various $\mathrm{CVH}$ metrics were measured by trained individuals instead of being based on self-reports.

In conclusion, in this population-based study on young adults, the prevalence of ideal CVH was $9.3 \%$. An inverse graded association was observed between ideal CVH score and CIMT, which also decreased and the probability of presenting a high cIMT by $32 \%$. Moreover, cIMT was significantly and inversely associated with each ideal glucose, ideal blood pressure, and ideal BMI. It is suggested that future studies 
with larger sample sizes; investigating the relationship between ideal CVH metrics and cIMT and other surrogate markers of subclinical atherosclerosis; are needed in the MENA region. It is also necessary to conduct longitudinal studies to evaluate cIMT changes over time and assess its relationship with ideal $\mathrm{CVH}$ metrics, considering the weight of each of the seven $\mathrm{CVH}$ metrics on cIMT.

\section{Declarations}

Ethical approval and consent to participate: Ethical approval for the TLGS study was obtained from the Ethics Committee of the Research Institute for Endocrine Sciences, Shahid Beheshti University of Medical Sciences. All the participants provided written informed consent. All the methods were carried out in accordance with relevant guidelines and regulations. Approval for undertaking the current project was also obtained from the Research Institute for Endocrine Sciences, Shahid Beheshti University of Medical Sciences, Tehran, Iran (IR.SBMU.MSP.REC.1399.759).

Consent for publication: Not Applicable.

Availability of data and materials: The datasets used and analyzed during the current study are available from the corresponding author on reasonable request.

Competing interest: The authors declare that they have no financial or non-financial competing interests.

Funding: None

\section{Authors' contributions:}

Study conception and design: VC, FH, FA, MN, PD, AMM, PM

Drafting and revising the article: $\mathrm{VC}, \mathrm{AE}, \mathrm{MB}, \mathrm{FH}, \mathrm{MV}$

Analysis and interpretation of data: MM

Final approval: $V C, F H, F A, M N, P D, A M M, P M, M M, A E, M B, F H, M V$

Acknowledgments: This article was the result of Vajihe Chavoshi's thesis. We have to express our sincere thanks to all the personnel of the Research Institute for Endocrine Sciences of Shahid Beheshti University of Medical Sciences (SBMU) for respectable cooperation in data collection.

\section{References}

1. Virani, S. S. et al. Heart Disease and Stroke Statistics-2020 Update: A Report From the American Heart Association., 141 (9), e139-e596 (2020).

2. (WHO) WHO. Cardiovascular diseases (CVDs): World Health Organization (WHO); 2017 [Available from: https://www.who.int/news-room/fact-sheets/detail/cardiovascular-diseases-(cvds) 
3. Lloyd-Jones, D. M. et al. Defining and setting national goals for cardiovascular health promotion and disease reduction: the American Heart Association's strategic Impact Goal through 2020 and beyond., 121 (4), 586-613 (2010).

4. Huffman, M. D. et al. Cardiovascular health behavior and health factor changes (1988-2008) and projections to 2020: results from the National Health and Nutrition Examination Surveys., 125 (21), 2595-2602 (2012).

5. Younus, A. et al. A Systematic Review of the Prevalence and Outcomes of Ideal Cardiovascular Health in US and Non-US Populations. Mayo Clinic proceedings. 2016;91(5):649-70.

6. Polonsky, T. S. et al. Association of Cardiovascular Health With Subclinical Disease and Incident Events: The Multi-Ethnic Study of Atherosclerosis.Journal of the American Heart Association. 2017;6(3).

7. Gaye, B. et al. Ideal Cardiovascular Health, Mortality, and Vascular Events in Elderly Subjects: The Three-City Study. Journal of the American College of Cardiology, 69 (25), 3015-3026 (2017).

8. Crichton, G. E., Elias, M. F. \& Robbins, M. A. Cardiovascular health and arterial stiffness: the MaineSyracuse Longitudinal Study. Journal of human hypertension, 28 (7), 444-449 (2014).

9. Plichart, M. et al. Carotid intima-media thickness in plaque-free site, carotid plaques and coronary heart disease risk prediction in older adults. The Three-City Study. Atherosclerosis, 219 (2), 917-924 (2011).

10. Lorenz, M. W., Markus, H. S., Bots, M. L., Rosvall, M. \& Sitzer, M. Prediction of clinical cardiovascular events with carotid intima-media thickness: a systematic review and meta-analysis., 115 (4), 459467 (2007).

11. Kablak-Ziembicka, A. et al. Association of increased carotid intima-media thickness with the extent of coronary artery disease., 90 (11), 1286-1290 (2004).

12. Touboul, P. J. et al. Mannheim carotid intima-media thickness and plaque consensus (2004-20062011). An update on behalf of the advisory board of the 3rd, 4th and 5th watching the risk symposia, at the 13th, 15th and 20th European Stroke Conferences, Mannheim, Germany, 2004, Brussels, Belgium, 2006, and Hamburg, Germany, 2011. Cerebrovascular diseases (Basel, Switzerland). 2012;34(4):290-6.

13. Nonterah, E. A. et al. Poor cardiovascular health is associated with subclinical atherosclerosis in apparently healthy sub-Saharan African populations: an H3Africa AWI-Gen study. BMC medicine, 19 (1), 30 (2021).

14. Oikonen, M. et al. Ideal cardiovascular health in young adult populations from the United States, Finland, and Australia and its association with cIMT: the International Childhood Cardiovascular Cohort Consortium. Journal of the American Heart Association, 2 (3), e000244 (2013).

15. Santos, I. S., Goulart, A. C., Pereira, A. C., Lotufo, P. A. \& Benseñor, I. M. Association between Cardiovascular Health Score and Carotid Intima-Media Thickness: Cross-Sectional Analysis of the Brazilian Longitudinal Study of Adult Health (ELSA-Brasil) Baseline Assessment. Journal of the 
American Society of Echocardiography: official publication of the American Society of Echocardiography, 29 (12), 1207-1164 (2016).

16. Shpilsky, D. et al. Association between ideal cardiovascular health and markers of subclinical cardiovascular disease. Clinical cardiology, 41 (12), 1593-1599 (2018).

17. Moghaddam, M. M. et al. Distribution of ideal cardiovascular health in a community-based cohort of Middle East population. Annals of Saudi medicine, 34 (2), 134-142 (2014).

18. Azizi, F. et al. Prevention of non-communicable disease in a population in nutrition transition: Tehran Lipid and Glucose Study phase II. Trials, 10, 5 (2009).

19. Koo, T. K. \& Li, M. Y. A Guideline of Selecting and Reporting Intraclass Correlation Coefficients for Reliability Research. Journal of chiropractic medicine, 15 (2), 155-163 (2016).

20. Esfahani, F. H., Asghari, G., Mirmiran, P. \& Azizi, F. Reproducibility and relative validity of food group intake in a food frequency questionnaire developed for the Tehran Lipid and Glucose Study. Journal of epidemiology, 20 (2), 150-158 (2010).

21. Momenan, A. A. et al. Reliability and validity of the Modifiable Activity Questionnaire (MAQ) in an Iranian urban adult population. Archives of Iranian medicine, 15 (5), 279-282 (2012).

22. Azizi, F. et al. Metabolic health in the Middle East and north Africa. The lancet Diabetes \& endocrinology, 7 (11), 866-879 (2019).

23. Peng, Y., Cao, S., Yao, Z. \& Wang, Z. Prevalence of the cardiovascular health status in adults: A systematic review and meta-analysis. Nutrition, metabolism, and cardiovascular diseases. NMCD, 28 (12), 1197-1207 (2018).

24. Rahmani, F. et al. National trends in cardiovascular health metrics among Iranian adults using results of three cross-sectional STEPwise approaches to surveillance surveys. Scientific reports, 11 (1), 58 (2021).

25. Wang, L. et al. Ideal Cardiovascular Health is Inversely Associated with Subclinical Atherosclerosis: A Prospective Analysis. Biomedical and environmental sciences: BES, 32 (4), 260-271 (2019).

\section{Figures}




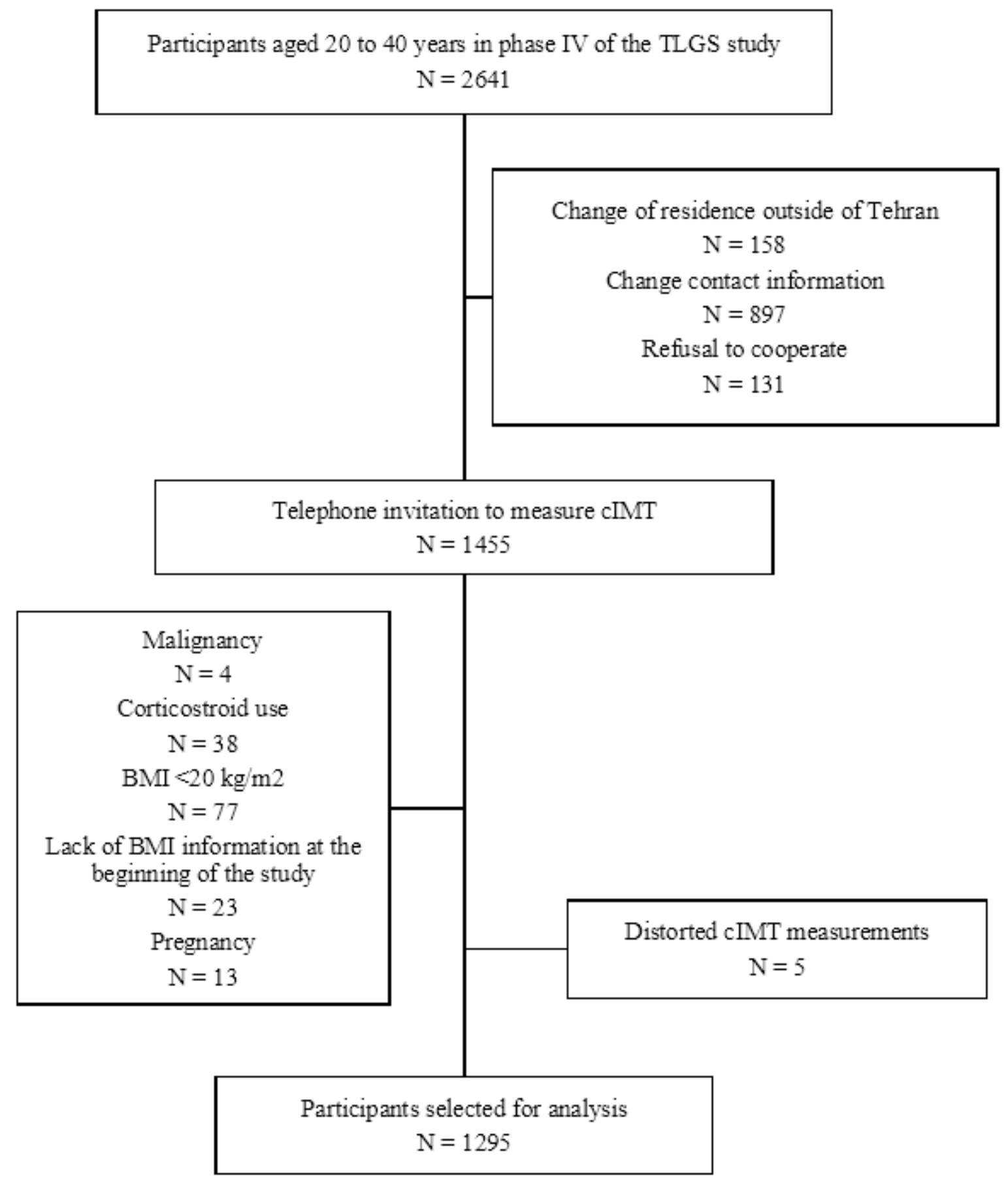

\section{Figure 1}

The flow chart of the study population. 


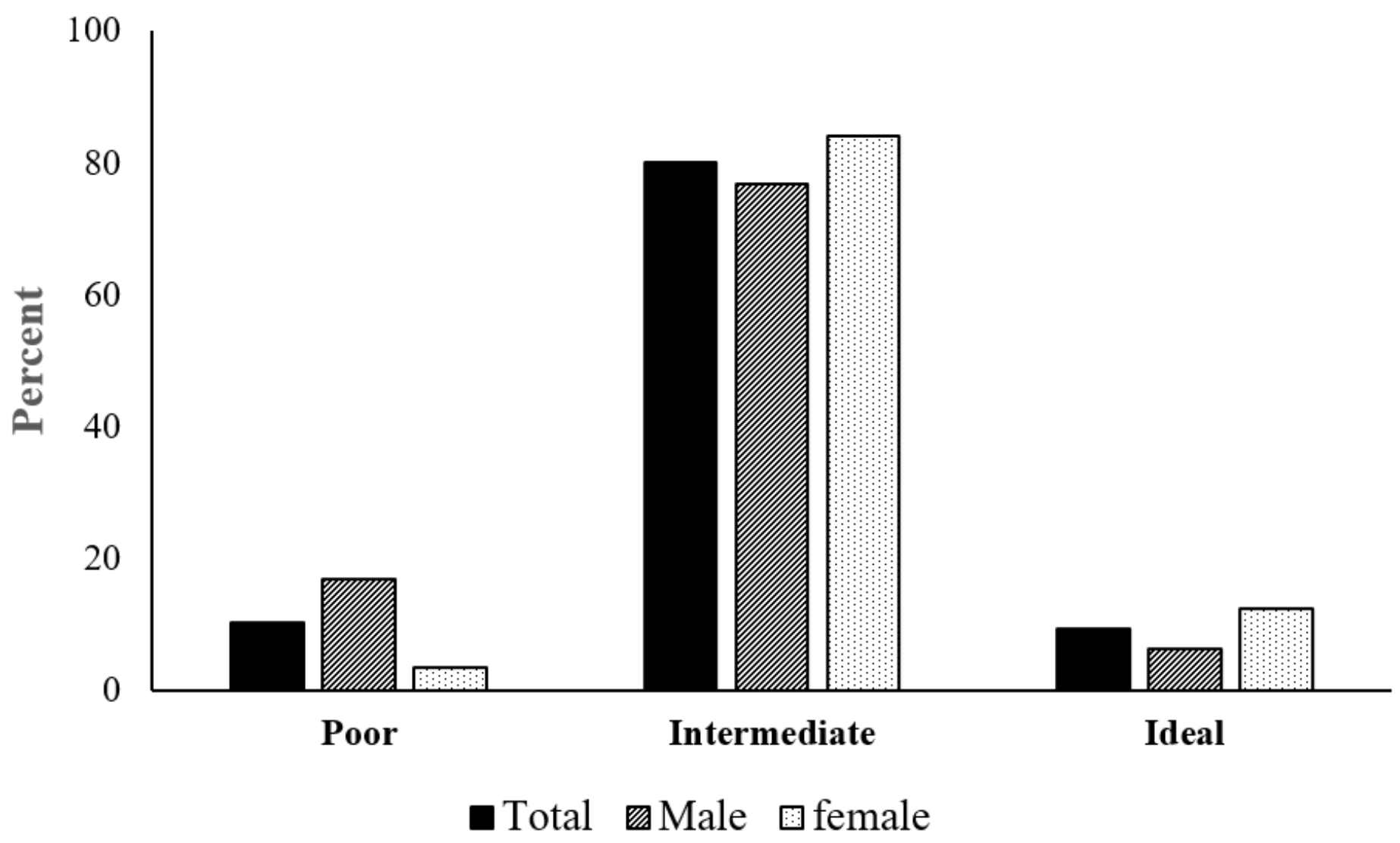

Figure 2

The distribution of study participants into three distinct CVH categories. 


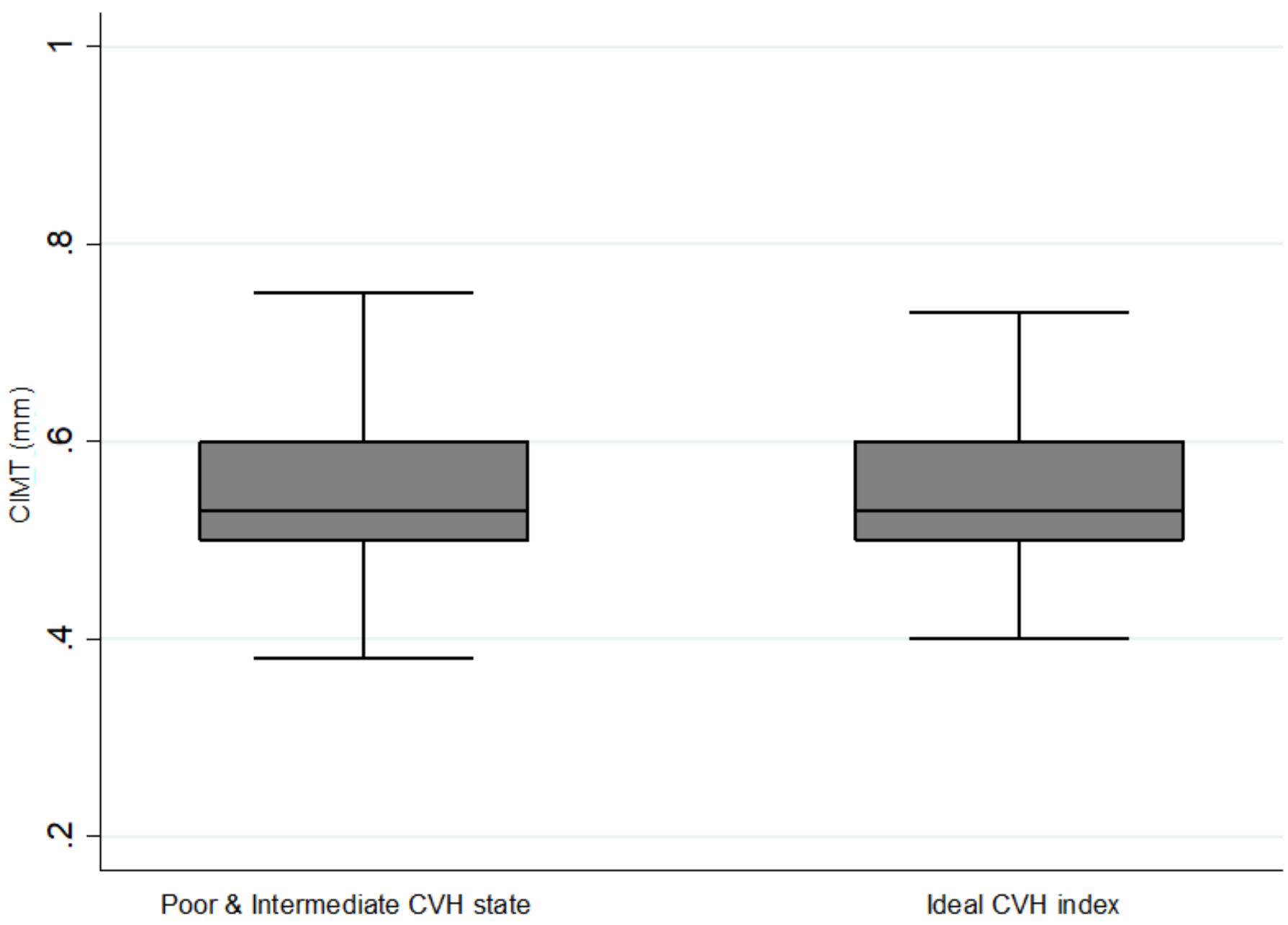

Figure 3

The box plots showing clMT in the two groups of ideal and poor/intermediate $\mathrm{CVH}$. 